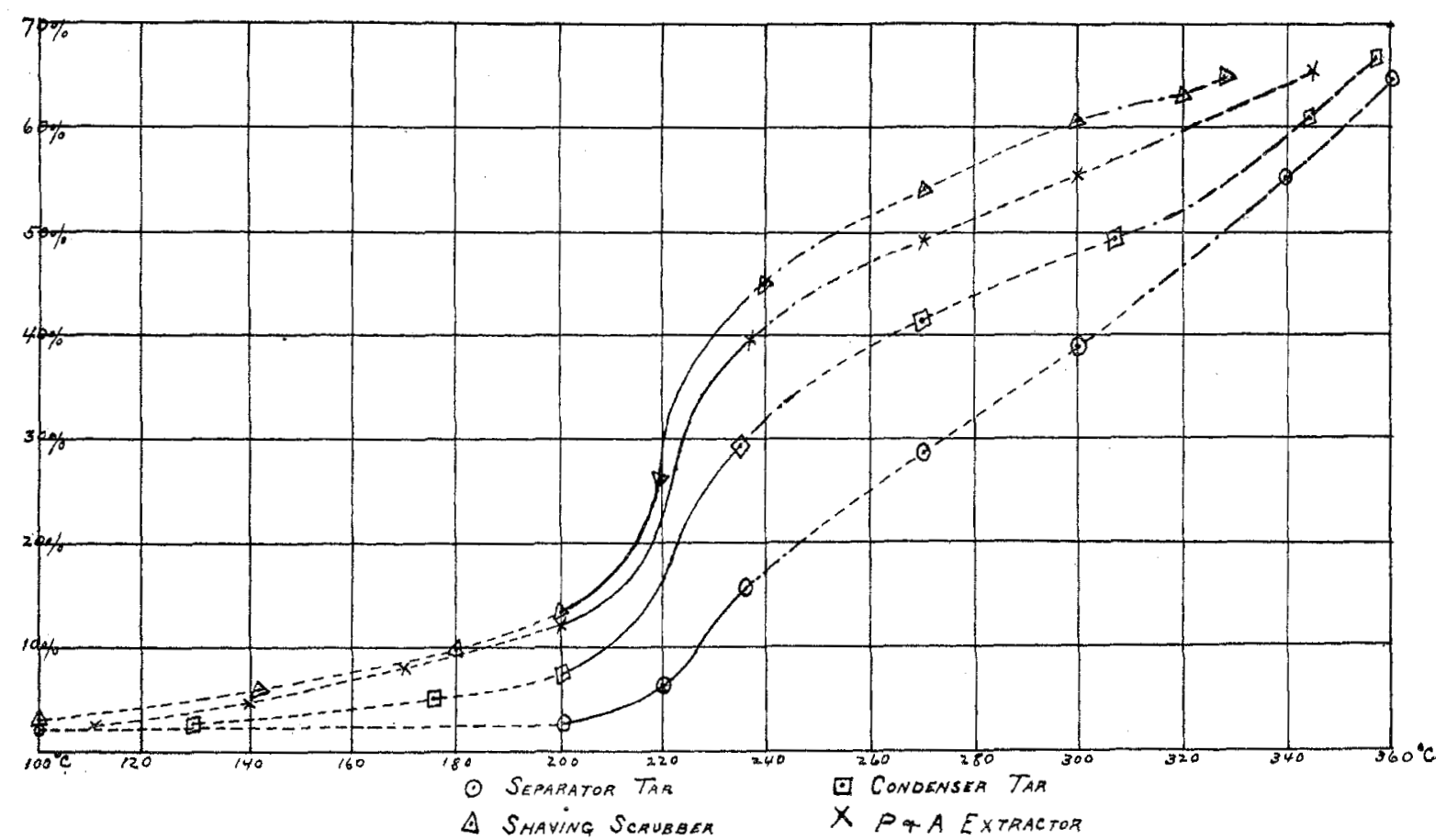

Fig. 2.

in which solid lines indicate distillates solidifying on cooling, dotted lines those remaining liquid, and broken lines the intermediate consistencies. As foreshadowed by the differences in specific gravity, the tars vary in the proportions of high-and low-boiling constituents, and become progressively lower-boiling from the separator tar to the shaving scrubber tar.

The fractions were particularly satisfactory in color and general character. The low-boiling liquid distillates were pale yellow, clear liquids, the naphthalene fractions almost pure white and solid at room temperatures, and the high-boiling portions of decidedly better color than the corresponding fractions from coal tar. There is a similarity in the general appearance of the distillates from coal tar and water gas tar, especially in the portions boiling below $300^{\circ} \mathrm{C}$. The high-boiling portions of the water gas tar are noticeably less solid than those from coal tar. In the case of the latter the distillate becomes decidedly hard as the temperature rises. Solid distillates are also obtained from the water gas tar above $300^{\circ} \mathrm{C}$., but there is a range between $340^{\circ}$ and $360^{\circ}$ where with a good fractionating device a clear, heavy liquid distillate is obtained, which after standing shows some crystals but does not become solid, a marked contrast to the distillate from coal tar at this point.

The differences between the distillates from the different samples of water gas tar which had separated at different points in the purifying system were apparently quantitative rather than qualitative, the distillates coming off from the different ones at the same temperatures being indistinguishable.

A. L. DEAN,

C. R. Downs.

Shrffield Chemical Laboratory, Yale UnIVERsity.

\section{NOTE ON THE DISTILLATION OF TARS CONTAINING WATER.}

Great difficulty is met with at times when it is necessary to distil a mixture of tar and water. Some gas tars contain as high as 75 per cent. of water in the form of an emulsion that will not break on long

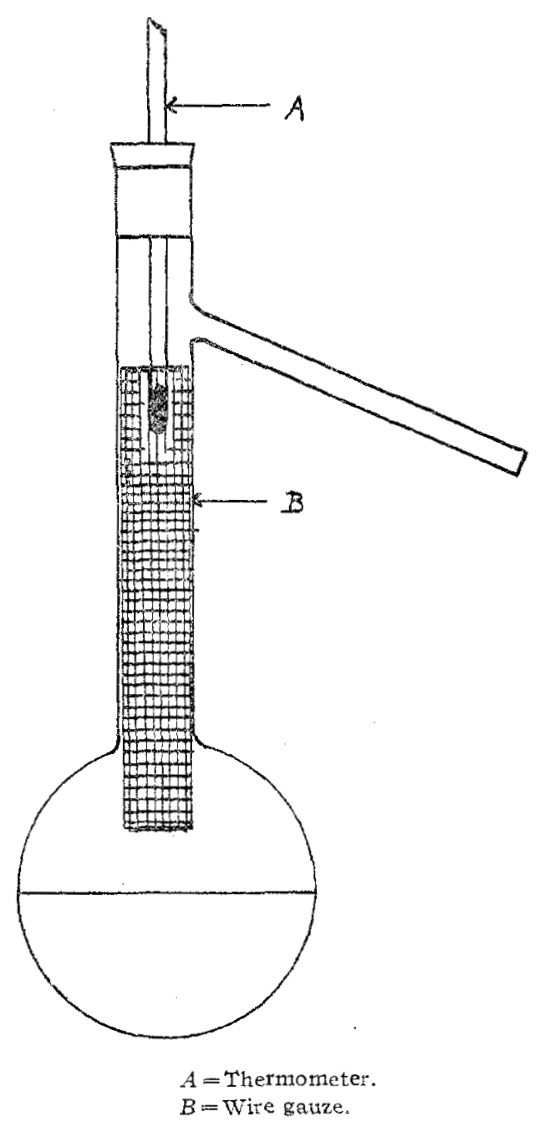


standing. If this emulsion is distilled from a retort or ordinary distilling bulb it is almost sure to "bump over," the water condensing on the thermometer or sides of the neck and dropping back into the heated tar, causing it to bump. This is prevented by making two concentric cylinders of " 30 mesh" brass gauze, which are slipped into the neck. This prevents any tar that does bump from going over at the beginning of the distillation. After the distillation has begun, the brass cylinders conduct heat upwards and any water collecting above is volatilized from the gauze instead of dropping back into the tar. There is plenty of room for the thermometer bulb inside the inner cylinder. Besides preventing bumping, the gauze cylinders act to some extent as a Hempel column and give a better service than the bare distilling bulb. The gauze is suspended by small wires that are held in place by the cork. C. R. Downs. SheFfield Sctentific School,

CHEMICAI LABORATORY.

\section{PRECIPITATION OF SULPHUR FROM DILUTE LIME-SUL- PHUR SOLUTIONS.}

A recent development in the use of the lime-sulphur solution for spraying purposes is as a summer fungicide, for which purpose it promises to supplant to a considerable extent the older Bordeaux mixture. The dilution is necessarily much greater than that used in the winter, since injury to the foliage would otherwise result, but as yet there is no unanimity of opinion among authorities as to the relative degree of this dilution, and sufficient data from practical results has not yet accumulated to permit of any positive conclusions.

The range of dilutions recommended in several publications is shown in Table 1 . For the sake of comparison, the original data has been recalculated to the basis of a density of I.30, assuming that the density of a solution varies inversely with the number of dilutions. This is not strictly true in the case of lime-sulphur solutions, but is accurate enough for the present purpose.

TABLE 1.

Gals. water added

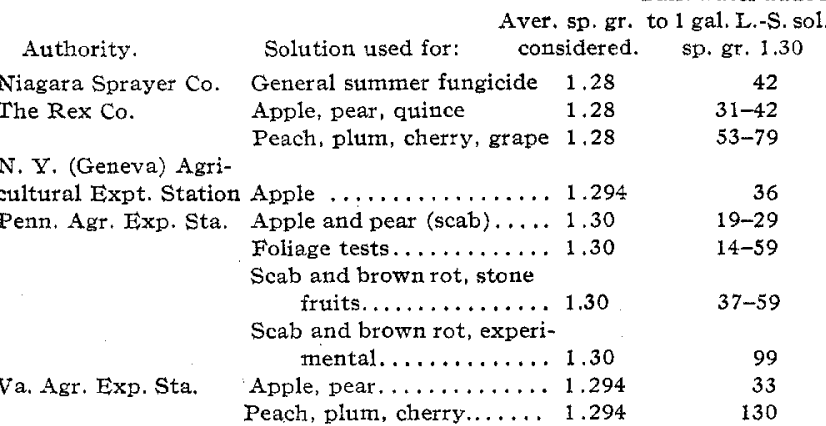

The minimum and maximum dilutions recommended are respectively $I_{4}$ and $I_{30}$ gallons of water to I gallon of $\mathrm{I} .30$ lime-sulphur solution. Practical results already secured would seem to indicate that in the case of apples a dilution of 30 gives a weak enough solution, but for more tender fruits a greater dilution will probably be necessary. There is, however, a factor entirely independent of spraying results which will be found to limit the dilution safely attainable, the precipitation of free sulphur by too great an excess of water.

While investigating the properties of concentrated lime-sulphur solutions, the writer noticed that a precipitate of sulphur quickly formed after running water into a beaker from which such a solution had just been poured. It appearing not improbable that this behavior might prove an important consideration in the dilution of solutions for spraying purposes, further attention was at once given it. The view is now quite generally held that the calcium-sulphur compounds, giving to the solution its principal value as an insecticide and fungicide, are the two higher sulphides $\mathrm{CaS}_{4}$ and $\mathrm{CaS}_{5}$. If by excessive dilution, these are broken up into free sulphur and various compounds, a direct loss of efficiency occurs, destroying, in a great measure, the value of the solution except insofar as a mixture of sulphur and water would be of benefit.

Qualitative tests showed that the precipitation took place slowly if comparatively small amounts of water were used for the dilution, but almost immediately if large volumes were introduced. The relative amount of sulphur deposited became greater as the dilution was increased, while the color of the precipitated sulphur ranged from orange to milk-white under the same conditions. There seemed to be no difference in results whether the solution was added to water or vice versa.

A short series of quantitative tests was then planned, in order to secure data for use in practical work. A solution was taken for dilution having a density of I.03977, it having been already diluted up from a concentrated solution. Six 5-cc. portions were pipetted into beakers containing appropriate volumes of water, the time of addition was noted, and the beakers were allowed to stand until sulphur was precipitated. The results are shown in Table 2 .

\begin{tabular}{|c|c|c|c|c|c|c|}
\hline No. & $\begin{array}{c}\text { Cc. water } \\
\text { added. }\end{array}$ & Added at: & $\begin{array}{l}\text { TABLE } \\
\text { Dil. from } \\
\text { sol. taken. }\end{array}$ & $\begin{array}{r}\text { Dil. from } \\
\text { sp. gr. } 1.30\end{array}$ & 'Time ppt'd. & Hours. \\
\hline 1 & 15 & $7.56 \mathrm{A.M}$ & 4 & 30.18 & - & - \\
\hline 2 & 25 & 7.57 A.M. & 6 & 45.26 & 一 & 一 \\
\hline 3 & 35 & 7.58 A.M. & 8 & 60.35 & - & - \\
\hline 4 & 50 & 7.59 A.M. & 11 & 82.98 & 1.30 P.M. & $5^{1 / 2}$ \\
\hline 5 & 80 & 9.14 A.M. & 17 & 128.25 & 11.30 A.M. & $21 / 4$ \\
\hline 6 & 150 & 9.24 A.M. & 37 & 233.90 & 9.35 A.M. & 11 \\
\hline
\end{tabular}

Upon prolonged standing even the first three developed traces of a precipitate, but this was probably due to atmospheric action and would have been prevented had the solutions been protected by a layer of oil. As was the case with the qualitative tests, the more dilute the solution the greater the relative precipitation of sulphur and the more quickly it was thrown down.

In order to ascertain whether this precipitated sulphur could be redissolved a trial was made with precipitation No. 5, boiling the solution and sulphur together for some time. Apparently about half of the sulphur dissolved, but long before the solution was cool it was reprecipitated. A second boiling failed to give a similar effect, the sulphur having apparently settled in an insoluble form upon cooling. 